\title{
Complementary detection of confined acoustic phonons in quantum dots by coherent phonon measurement and Raman scattering
}

\author{
Michio Ikezawa, Tsuyoshi Okuno, and Yasuaki Masumoto \\ Institute of Physics, University of Tsukuba, Tsukuba 305-8571, Japan
}

Andrey A. Lipovskii

St.-Petersburg State Technical University, Polytekhnicheskaja 29, St.-Petersburg, 195251 Russia

(Received 11 July 2001; revised manuscript received 17 September 2001; published 2 November 2001)

\begin{abstract}
Coherent acoustic phonon oscillation is observed in PbSe quantum dots embedded in phosphate glass by femtosecond pump-and-probe. The size dependence of the oscillation is investigated. Distinct low-frequency peaks are observed in Raman spectrum for the same samples. The size-dependence of the frequencies is well explained by elastic sphere model, but the observed modes are different to each other for coherent phonon and Raman scattering. Coherent phonon measurement and Raman scattering are found to give complementary information on confined acoustic phonons in this system.
\end{abstract}

DOI: 10.1103/PhysRevB.64.201315

PACS number(s): 63.22. $+\mathrm{m}, 78.47 .+\mathrm{p}$

Semiconductor quantum dots (QDs) have attracted considerable attention in recent years. ${ }^{1,2}$ Three-dimensional confinement of electrons to a nanometer-size space changes their continuous energy spectrum to discrete one. It not only modifies electronic state, but also modifies phonon spectrum to size-dependent discrete lines, and the electron-phonon interaction among them is thought to be changed also. The size-dependence of the exciton-phonon interaction in quantum dot has been the subject of extensive research. Experimental studies on the optical, ${ }^{3,4}$ or acoustic ${ }^{5-8}$ phonon modes in QDs have been carried out mainly by Raman scattering. Persistent spectral hole-burning (PSHB) spectroscopy was also successfully applied to QDs. ${ }^{9,10}$ During the last decade, coherent oscillation of the confined vibrational modes became to be observable directly in time domain. There are some reports on the observation of coherent optical phonons in semiconductor QDs, CdSe QDs, ${ }^{11}$ InP QDs, ${ }^{12}$ etc. While the high energy optical phonon forms sidebands in optical spectrum, low energy acoustic phonon rather contributes to excitonic dephasing and homogeneous broadening, ${ }^{13}$ which plays a crucial role in nonlinear device applications. Therefore a detailed understanding of the mechanism of generation and damping of confined acoustic phonon in QDs is important. Coherent oscillation of confined acoustic phonon in QDs was first reported by Krauss and Wise in $1997 .{ }^{14}$ They observed temporal oscillation of the transmission in femtosecond pump-and-probe measurement on $3 \mathrm{~nm}$ PbS QDs dispersed in PVA (polyvinyl alcohol). They also measured Raman scattering of the same sample to compare with coherent phonon. However, as they mentioned in the paper, they were not able to systematically study the size-dependence, because size control in the polymer matrix was difficult. Therefore, the size-dependence of the relation between coherent acoustic phonon and Raman spectrum has not been not studied yet. In this Rapid Communication, we detect coherent acoustic phonon signal in QDs embedded in glass matrix with high sensitivity. We measured size-dependence of coherent phonon and Raman scattering. The complementary relation of these measurements was clearly shown.
For coherent phonon measurements, we performed femtosecond pump-and-probe. A mode-locked Ti:sapphire oscillator was used to produce pump and probe pulses, whose pulse width was about $30 \mathrm{fs}$. Center wavelength of the near transform-limited pulse was about $790 \mathrm{~nm}$. A shaker was introduced into the pump beam path to modulate pump delay time at $80 \mathrm{~Hz}$. The pump and probe beams were focused on the sample surface, and the transmitted probe beam were detected together with a reference beam by a pair of Si photodiodes to cancel the instability of the laser. The subtracted signal was led to a lock-in amplifier. To measure Raman spectra, a cw-Ti:sapphire laser pumped by an Ar-ion laser was used as an excitation source. A 1-m double monochromator with a photon counting system was used for detection. Samples used in this study were PbSe QDs grown in phosphate glass matrix. ${ }^{15}$ The mean radius of all samples were estimated by Small-Angle X-ray Scattering (SAXS), and found to range between $1.4 \mathrm{~nm}$ and $3.5 \mathrm{~nm}$. All experiments in this study were performed at room temperature.

An absorption spectrum of the sample with mean radius $2.9 \mathrm{~nm}$ is shown in Fig. 1(a). The time trace of pump-andprobe measurement is shown in Fig. 1(b). This is an expanded curve at the tail part of a large absorption change. A clear oscillatory component can be recognized superimposed on the absorption change of excitons. The oscillation period is about $2 \mathrm{ps}$, and the amplitude rapidly damps. We note that we detected coherent phonon signal by using a small pump delay modulation and lock-in technique, and that the data obtained here corresponds to the time derivative of ordinary transmission intensity. Therefore the signal in Fig. 1(b) can be fitted by a damped sine function, but it means that the corresponding phonon oscillation is expressed by a damped cosine function. The amplitude of the oscillation is in an order of $10^{-6}$ times the total probe intensity. In contrast, in QDs embedded in polymer matrix, $\mathrm{PbS}$ in PVA, ${ }^{14}$ the modulation of probe intensity by the coherent confined acoustic phonon measured by time-resolved pump-and-probe was as large as $10^{-3}$ under almost equal excitation density to our experiment. It should be added that they did not observe coherent phonon signal in $\mathrm{PbS}$ in silicate glass matrix. It may 

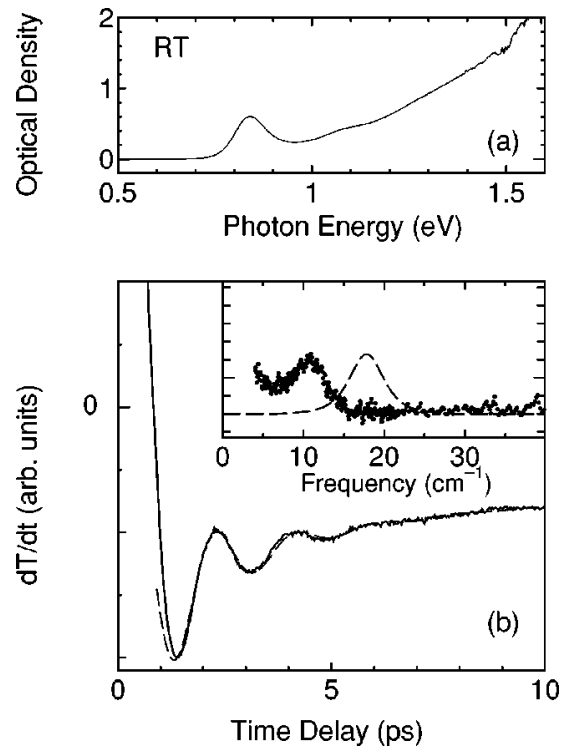

FIG. 1. (a) Absorption spectrum of PbSe QDs at RT. The mean radius is $2.9 \mathrm{~nm}$. (b) Time-resolved transmission signal of the same sample at RT. An overdamped oscillation due to confined acoustic phonon is clearly observed. A dashed line represents the fitting by a damped sine function. In the inset, dots correspond to the Raman spectrum of the same sample. A dashed line is the Fourier transformed spectrum of the fitting curve for the coherent phonon signal.

be due to the weakness of the signal in glass matrix. Small modulation signal due to coherent acoustic phonon in QDs in glass matrix was also observed on PbTe QDs in glass. ${ }^{16}$ This coherent phonon signal can be temporarily assigned to the confined acoustic phonon in the QDs because of its small frequency. Since the photon energy of the pump pulse is well above the first excited state of the sample, the pump pulses coherently excite all of QDs with various sizes in the sizedistribution. Therefore a lot of QDs having different phonon frequencies contribute to the coherent phonon signal. The damping does not mean the real damping of the coherent oscillation of the confined phonon in each quantum dot, but dephasing due to the inhomogeneous broadening of the phonon frequency. As shown later, the damping constant is reasonably explained by the size-distribution. Figure 2 shows the size-dependence of pump-and-probe signal. The oscilla-

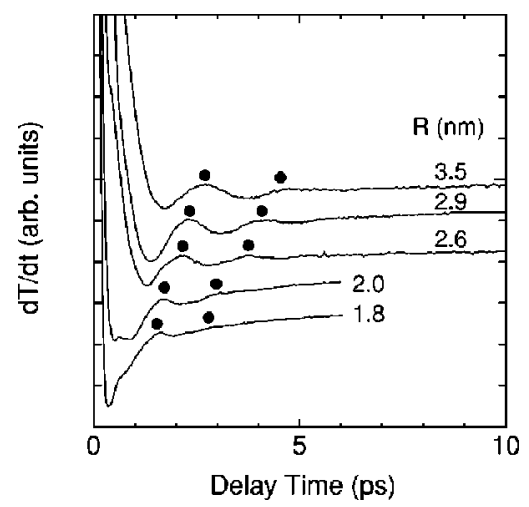

FIG. 2. Size-dependence of the coherent phonon signal. $\mathrm{R}$ is the mean radius of the $\mathrm{PbSe}$ QDs.

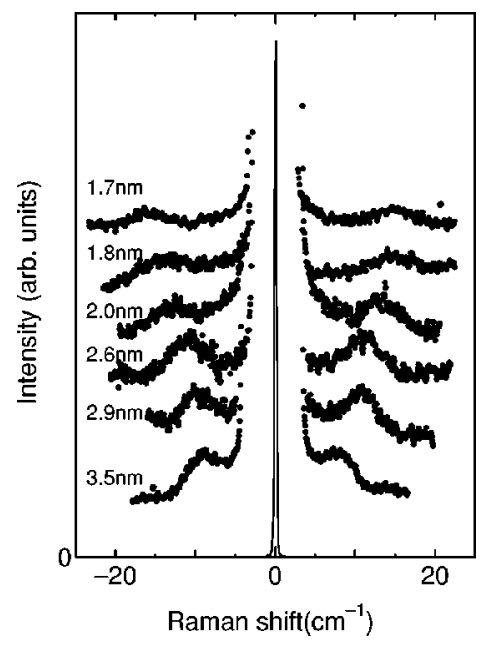

FIG. 3. Raman spectra for various size PbSe QDs at RT. Excitation laser was $750 \mathrm{~nm}$. Size-dependent peaks were observed around $10 \mathrm{~cm}^{-1}$.

tion frequency increases with decreasing dot radius. It supports the assignment of the oscillation to the confined acoustic phonon whose frequency is known to be inversely proportional to the dot radius as seen later. Since the oscillatory component is weaker than the background due to the fast recovery of absorption change, it is difficult to separate phonon signal from the background. Therefore we obtained size-dependent frequency from the interval between the first and the second hump marked by a solid circle in the figure.

To compare the coherent phonon with Raman scattering, Raman spectrum of $2.9 \mathrm{~nm}$ PbSe dots was measured by using $750 \mathrm{~nm}$ excitation laser. The results are displayed in the inset of Fig. 1(b) by dots. Fourier transformed spectrum of the fitting curve of the coherent phonon signal is also drawn in the inset of Fig. 1(b) by a dashed line. The peak position in the Raman spectrum is $11 \mathrm{~cm}^{-1}$ and it does not coincide with the spectrum of the coherent phonon signal. There is no structure at $18 \mathrm{~cm}^{-1}$ in the Raman spectrum. The sizedependence of Raman spectrum is shown in Fig. 3. The excitation laser was $750 \mathrm{~nm}$. The spectral resolution was $1.4 \mathrm{~cm}^{-1}$. Size-dependent low frequency peaks are clearly observed in the range from $8 \mathrm{~cm}^{-1}$ to $16 \mathrm{~cm}^{-1}$. With decreasing the dot size, the peak frequency increases and its width also increases, reflecting that the smaller dots have larger size-distribution. When we tried using an Ar-ion laser $(514.5 \mathrm{~nm})$ for excitation, the data was almost the same as $750 \mathrm{~nm}$ excitation. The peak frequencies in the Raman spectra are plotted as a function of dot radius by open circles in Fig. 4. For low frequency Raman spectrum, correction of the occupation number by Bose factor is needed in order to evaluate real phonon spectrum. The correction modifies the spectrum particularly in the very low frequency region, but does not strongly affect the peak position compared to the error bar under our experimental condition even for the largest dots. Solid circles in Fig. 4 represent the frequencies of the oscillation observed in the coherent phonon measurement. We can see the linear dependence of both frequencies on the inverse radius of dots, but the coherent phonon signal and the Raman signal seem to come from different confined 


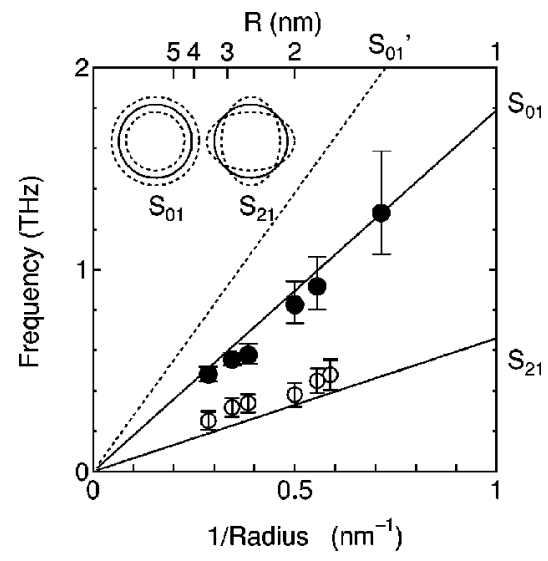

FIG. 4. Frequency of the coherent phonon and the Raman peak as a function of dot size. They are inversely proportional to the dot radius. Solid lines represent the calculation on the assumption that the stress at the dot boundary is zero, while a dashed line represents the calculation with rigid boundary condition. The inset represents the displacement in $\mathrm{S}_{01}$ and $\mathrm{S}_{21}$ spheroidal mode.

phonon modes in QDs. As is well known, vibration modes of a sphere with stress-free boundary condition were studied theoretically by Lamb. ${ }^{17}$ According to their results, the vibrational mode is classified into two categories, spheroidal modes and torsional modes. Duval ${ }^{18}$ derived the selection rule from the group theory that the mode observed in the first order Raman scattering was only spheroidal mode with $l$ $=0$ or $l=2$. The lowest mode of $l=0$ spheroidal mode is known as breathing mode, where expansion and shrinkage of whole sphere occur. This mode is purely longitudinal, but $l$ $=2$ is a mixed mode and have both longitudinal and transverse components. Displacements in these modes are illustrated in the inset of Fig. 4.

The frequencies of the spheroidal modes are expressed by $\nu_{l n}=\xi_{l n} V_{l} / R$, where $\xi_{l n}$ is a coefficient depending on the ratio of longitudinal $\left(V_{l}\right)$ and transverse $\left(V_{t}\right)$ sound velocities, and $R$ is the dot radius. ${ }^{19}$ If we assume stress-free boundary condition, the numerator of the expression can be calculated by using bulk $\mathrm{PbSe}$ parameters. Finally, for the lowest spheroidal mode with $l=0$, the numerator, $S_{l n}$ $=\xi_{l n} V_{l}$, is $\mathrm{S}_{01}=1.79$, that is $\nu_{01}(\mathrm{THz})=1.79 / R(\mathrm{~nm})$. We can also try to assume rigid boundary condition where the lattice displacement at boundary is zero. This assumption was applied for the interpretation of the Raman spectrum on CdSe nanocrystals in glasses. ${ }^{20}$ Under this assumption, the frequency of the spheroidal mode is shifted to higher frequency, $S_{01}^{\prime}=2.76$. They are represented in Fig. 4 labeled $S_{01}$ and $S_{01}^{\prime}$, respectively. The experimental data of the coherent phonon is well fitted by calculation based on the stress-free boundary condition. The assignment is supported by the phase of the observed coherent phonon signal. In coherent phonon measurement, the oscillation found to show cosinelike temporal behavior. In other words, the phonon amplitude is maximum at $t=0$ indicating the origin of instantaneous expansion by pump pulses. An instantaneous isotropic expansion occurs by the high density carriers impulsively excited by the femtosecond pump pulses, and the breathing mode oscillation starts. The oscillation modifies the optical spectrum of QDs through deformation potential. It causes small energy shift of the absorption spectrum synchronized with the oscillation, which led to the temporal change of the probe transmission we detected. On the other hand, the Raman peaks are assigned to $\mathrm{S}_{21}$ mode. It is the lowest $l=2$ spheroidal mode. $\mathrm{S}_{21}=0.66$ is deduced by assuming stressfree boundary condition. Small discrepancy may come from the fact that real $\mathrm{PbSe}$ is not exactly isotropic. The Raman active spheroidal modes $(l=0$ and $l=2)$ can be distinguished by their polarization dependence. ${ }^{18} l=0$ mode can be observed only in the same polarization as the excitation polarization, while $l=2$ mode can be also observed in cross polarization. We examined polarization dependence of the low-frequency peak in Raman spectrum. The peak was observed under both parallel and cross-polarization detection. Therefore it is acceptable to assign these peaks to $l=2$ mode.

In this way, based on their size-dependence, the observed Raman peaks and the frequencies of coherent phonon are unambiguously assigned to the different phonon modes. In other semiconductor quantum dots, for example, CdSe $\mathrm{QDs}^{20}$ and CdS QDs, ${ }^{8}$ both $l=0$ and $l=2$ acoustic modes were observed in Raman spectrum unlike our results. The point is that in PbSe QDs the Raman scattering cannot detect the breathing mode, on the contrary, coherent phonon measurement mainly detects the breathing mode and could be useful as a complementary detection technique. We can find experimental results similar to ours in the research field of metal nanoparticles. The observed Raman peaks of acoustic phonon of Ag nanoparticles is mainly the $l=2$ mode, ${ }^{21}$ while the observed coherent phonon oscillation corresponds to the $l=0$ mode. $^{22}$ Montagna and Dusi ${ }^{23}$ deduced that the $l=0$ mode was not Raman active in nanoparticles with cubic Bravais lattice and for the (dipole-induced-dipole DID) scattering mechanism and explained why the $l=2$ mode dominated the Raman spectra of Ag nanoparticles. Since PbSe has $\mathrm{NaCl}$-type crystal structure unlike $\mathrm{CdS}$ or $\mathrm{CdSe}$ where the crystal lattice is hexagonal, the same arguments as Ag nanoparticles may be applicable to the Raman spectra of $\mathrm{PbSe}$ QDs.

Let us explain the width of the power spectrum of the coherent phonon and Raman spectrum in the inset of Fig. 1(b). The size-distribution of the sample $(2.9 \mathrm{~nm})$ is estimated to be about $28 \%$ by the absorption spectrum in Fig. 1(a) and the relation between size and the first excited state energy which was previously reported. ${ }^{24}$ The sizedistribution also agrees to the estimation from SAXS data. It corresponds to a FWHM of $6 \mathrm{~cm}^{-1}$ for $\mathrm{S}_{01}$ mode and $2.9 \mathrm{~cm}^{-1}$ for $S_{21}$ mode, and they reasonably agree with the observed width of $8 \mathrm{~cm}^{-1}$ and $5.5 \mathrm{~cm}^{-1}$.

The reason why the coherent phonon signal of QDs in glass is smaller than that of QD embedded in polymer matrix was not clarified. We cannot simply state that, compared to polymers, the hardness of the glass matrix surrounding QDs prevents them from vibrating acoustically and led to the small amplitude of the coherent phonon, because, as stated before, the stress-free coherent oscillation of QDs in glass was observed in our study. Therefore the combination of the QDs and glass should be relatively weak. 
In Ref. 25, the $68 \mathrm{~cm}^{-1}$ Raman peak on $2 \mathrm{~nm}$ PbS QDs in PVA was assigned to confined acoustic phonon mode with $l=0 . \mathrm{PbS}$ is not so different from $\mathrm{PbSe}$ in elastic properties, but the frequency of the confined acoustic phonon is much larger. Moreover the size-dependence of the mode seems to be quite small, compared to the Raman spectrum on $3 \mathrm{~nm}$ $\mathrm{PbS}$ QDs, ${ }^{14}$ and unlike our results, the coherent phonon signal reproduces the Raman spectrum in respect of the acoustic mode in this system. Thus we see that there are still more problems on the acoustic vibration of QDs in host matrix.

In conclusion, size-dependence of the confined acoustic phonon modes in $\mathrm{PbSe}$ QDs embedded in glass matrix was investigated by coherent phonon measurement and Raman scattering. The size-dependent frequency is well explained by stress-free elastic sphere model. It was found that coherent phonon experiment measures different confined acoustic modes from that in Raman scattering and is a useful tool to study confined phonon in QDs.

This work was supported by the Ministry of Education, Science, Sports and Culture, Grant-in-Aid for Encouragement of Young Scientists, 12740170, 2000, "Research for the Future" Program JSPS-RFTF 97P00106 from the Promotion of Science, project research of University of Tsukuba, Kurata Foundation (Japan), and INTAS foundation (EC). Authors are thankful to Dr. T. Mishina for fruitful discussion.
${ }^{1}$ A.D. Yoffe, Adv. Phys. 42, 173 (1993).

${ }^{2}$ U. Woggon, Optical Properties of Semiconductor Quantum Dots (Springer, Berlin, 1997).

${ }^{3}$ M. Fujii, S. Hayashi, and K. Yamamoto, Appl. Phys. Lett. 57, 2692 (1990).

${ }^{4}$ A. Tanaka, S. Onari, and T. Arai, Phys. Rev. B 45, 6587 (1992).

${ }^{5}$ E. Duval, A. Boukenter, and B. Champagnon, Phys. Rev. Lett. 56, 2052 (1986).

${ }^{6}$ N.N. Ovsyuk, E.B. Gorokhov, V.V. Grishchenko, and A.P. Shebanin, JETP Lett. 47, 298 (1988).

${ }^{7}$ B. Champagnon, B. Andrianasolo, and E. Duval, J. Chem. Phys. 94, 5237 (1991).

${ }^{8}$ A. Tanaka, S. Onari, and T. Arai, Phys. Rev. B 47, 1237 (1993).

${ }^{9}$ S. Okamoto and Y. Masumoto, J. Lumin. 64, 253 (1995).

${ }^{10}$ J. Zhao and Y. Masumoto, Phys. Rev. B 60, 4481 (1999); J. Zhao, S.V. Nair, and Y. Masumoto, ibid. 63, 033307 (2001).

${ }^{11}$ R.W. Schoenlein, D.M. Mittleman, J.J. Shiang, A.P. Alivisatos, and C.V. Shank, Phys. Rev. Lett. 70, 1014 (1993).

${ }^{12}$ U. Banin, G. Cerullo, A.A. Guzelian, C.J. Bardeen, A.P. Alivisatos, and C.V. Shank, Phys. Rev. B 55, 7059 (1997).

${ }^{13}$ M. Ikezawa and Y. Masumoto, Phys. Rev. B 61, 12662 (2000).

${ }^{14}$ T.D. Krauss and F.W. Wise, Phys. Rev. Lett. 79, 5102 (1997).
${ }^{15}$ A.A. Lipovskii, E.V. Kolobkova, and V.D. Petrikov, Electron. Lett. 33, 101 (1997); A. Lipovskii, E. Kolobkova, V. Petrikov, I. Kang, A. Olkhovets, T. Krauss, M. Thomas, J. Silcox, F. Wise, Q. Shen, and S. Kycia, Appl. Phys. Lett. 71, 3406 (1997).

${ }^{16}$ E.R. Thoen, G. Steinmeyer, P. Langlois, E.P. Ippen, G.E. Tudury, C.H. Brito Cruz, L.C. Barbosa, and C.L. Cesar, Appl. Phys. Lett. 73, 2149 (1998).

${ }^{17}$ H. Lamb, Proc. London Math. Soc. 13, 51 (1882).

${ }^{18}$ E. Duval, Phys. Rev. B 46, 5795 (1992).

${ }^{19}$ B. A. Alud, Acoustic Fields and Waves in Solids (Wiley, New York, 1973).

${ }^{20}$ L. Saviot, B. Champagnon, E. Duval, I.A. Kudriavtsev, and A.I. Ekimov, J. Non-Cryst. Solids 197, 238 (1996).

${ }^{21}$ G. Mariotto, M. Montagna, G. Viliani, E. Duval, S. Lefrant, E. Rzepka, and C. Mai, Europhys. Lett. 6, 239 (1988).

${ }^{22}$ N. Del Fatti, C. Voisin, F. Chevy, F. Vallée, and C. Flytzanis, J. Chem. Phys. 110, 11484 (1999).

${ }^{23}$ M. Montagna and R. Dusi, Phys. Rev. B 52, 10080 (1995).

${ }^{24}$ T. Okuno, Y. Masumoto, M. Ikezawa, T. Ogawa, and A.A. Lipovskii, Appl. Phys. Lett. 77, 504 (2000).

${ }^{25}$ T.D. Krauss, F.W. Wise, and D.B. Tanner, Phys. Rev. Lett. 76, 1376 (1996). 